\title{
Oblique incidence and observation electronic speckle-pattern interferometry
}

\author{
C. Joenathan, B. Franze, and H. J. Tiziani
}

\begin{abstract}
In this paper we discuss an oblique incidence and observation electronic speckle-pattern interferometer, in which we use an anamorphic prism in front of the object. A collimated beam traveling through the prism is partly reflected at the base of the prism. The reflected light is the reference beam and the transmitted light illuminates the diffuse object, thereby generating the object beam. In this scheme the object and the reference beams are collinear. A new scheme that uses two prisms and permits phase stepping is also presented.

Key words: Speckle interferometry, electronic speckle-pattern interferometer.
\end{abstract}

\section{Introduction}

Electronic speckle-pattern interferometry (ESPI) is a well-established tool for measuring out-of-plane and in-plane displacements of diffuse objects. ${ }^{1-3}$ Usually the observation direction in conventional ESPI systems is normal to the object, thereby providing one-to-one correspondence between an image point and an object point. On one hand, this scheme makes the system sensitive to out-of-plane motion in which each fringe corresponds to $\lambda / 2$ displacement. On the other hand, the sensitivity can be reduced by oblique incidence and observation, but the disadvantage of oblique observation is the anamorphic distortion of the object. Therefore the sensitivity can be varied only to a small extent by an increase in the angle of illumination. In addition, with the oblique angle of illumination the system becomes sensitive to in-plane motion as well, thereby making analysis difficult.

In some applications such as nondestructive testing or nondestructive evaluation low sensitivity is required to facilitate large-object deformation. This is possible if oblique incidence and observation are used. In this paper we propose an oblique incidence and

\footnotetext{
When this research was performed the authors were with the Institut fuer Technische Optik, Universitaet Stuttgart, Postfach 801140,70511 Stuttgart, Germany. C. Joenathan is now with the Department of Physics and Applied Optics, Rose Hulman Institute of Technology, 5500 Wabash Avenue, Terre Haute, Indiana 47803.

Received 25 August 1993; received revised manuscript 20 April 1994.

0003-6935/94/317307-05\$06.00/0.

- 1994 Optical Society of America.
}

observation ESPI in which we use an anamorphic (three-corner prism) or a Dove prism in front of the object. These prisms have also been used in specular interferometers to observe fringes from rough surfaces because of the oblique incidence and observation. 4-7 Unlike interferomtery, in ESPI the object surface scatters light and has to be imaged onto a recording medium. In our setup the medium of recording is a CCD camera, and the electronic signal is digitized and processed by a frame-grabber board in a host computer. Further, in ESPI the ratio of the reference to object beam has to be adjusted to obtain a tolerable signal-to-noise ratio. ${ }^{8}$ We accomplish this by using a polarized laser beam to illuminate the object and a polarizer placed in front of the camera. In our arrangement the reference beam that is collinear to the object beam is generated from the prism. In this regard, the proposed ESPI setup is similar to the quasi-equal path ESPI system. ${ }^{9,11}$ For oblique incidence and observation we used a right-angle prism. However, the analysis presented here is applicable to other prisms as well. With a regular threecorner prism the restriction on the distance of the object to the base side of the prism is also analyzed. A method to overcome this drawback is also discussed. This method also permits phase stepping in the reference beam. Results with phase stepping are also presented.

\section{Experimental Setup}

The light from a laser source is expanded with a microscope objective and is collimated by lens $L_{1}$ as shown in Fig. 1. This beam is then directed to a right-angle prism at angle $\theta_{1}$. This angle is chosen such that the angle of the beam incident within the 


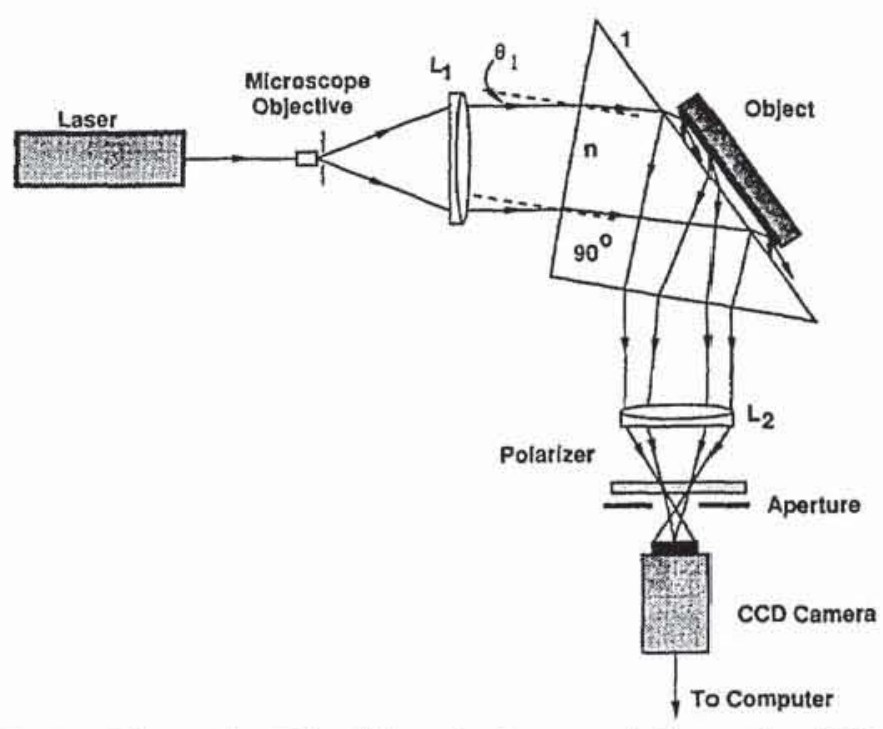

Fig. 1. Schematic of the oblique incidence and observation ESPI system. The reflection from the base side of the prism is used to generate the reference beam.

prism on surface 1 is smaller than the critical angle. Part of the beam is reflected at surface 1 and serves as the reference beam. The transmitted beam illuminates the diffuse object placed close to the prism. Achromat $\mathrm{L}_{2}$ is used to image the object onto the plane of the photosensor of the CCD camera. The reference beam, a nominally collimated beam is focused by $\mathrm{L}_{2}$ near the focal plane of the achromat. (In our setup the reference beam is slightly converging and its effect is discussed in the theory section.) A small aperture, shown in Fig. 1, is placed in this plane to control the size of the speckle and to permit the reference beam to pass through. To control the intensity of the reference beam, we place a rotatable polarizer near the aperture. As the object is coated with matte spray powder the scattered light is unpolarized. Therefore, the intensity of the object beam is not modified as the polarizer is rotated, ${ }^{9,10}$ allowing for a wide range of reference-to-object beam ratios. A reference-to-object beam ratio of $3: 1$ was used in our experiments. No aberration is introduced in the object illumination beam if the light entering the prism is collimated. However, with a converging beam, the object illumination beam becomes highly astigmatic. Because the initial and final frames (i.e., before and after deformation of the object) are subtracted, this aberration is canceled out and is of no interest to us. As the object scatters light, each scattering center can be assumed to be a point source that generates spherical waves. These cones of rays, after traversing through the other half of the prism, are astigmatic. This is a major concern in this interferometric arrangement. By using an aperture as discussed before, we greatly reduced astigmatism. Good contrast fringes were observed, usually with small apertures.

\section{Theory}

The ray diagram for a three-corner prism is shown in Fig. 2. If $L_{0}$ is the diameter of the collimated beam entering the prism, then the beam is stretched along one axis and has the shape of an ellipse. The length of the minor axis, $L_{\mathrm{mi}}$, is $L_{0}$, and that of the major axis is

$$
L_{\mathrm{ma}}=\frac{L_{0} \cos \theta_{2}}{\cos \theta_{1} \cos \left(\theta_{2}-A / 2\right)}=\frac{L_{0}}{\epsilon},
$$

where

$$
\epsilon=\frac{\cos \theta_{1} \cos \left(\theta_{2}-A / 2\right)}{\cos \theta_{2}},
$$

$A$ is the apex angle of the prism, and $\theta_{2}$ is the angle of refraction of the beam at the first interface. The angle of illumination in terms of the angle of the beam entering the prism is

$$
\theta_{3}=\sin ^{-1}\left\{n \sin \left[\frac{\pi}{2}-\frac{A}{2}-\sin ^{-1}\left(\frac{\sin \theta_{1}}{n}\right)\right]\right\},
$$

where $n$ is the refractive index of the prism. If a glass plate is used instead of a prism then the image of a circular object will appear like an ellipse, with large eccentricity. If $R$ is the radius of the circle then the major axis remains $R$, whereas the minor axis of the ellipse becomes $R \cos \theta_{3}$. However, with a prism this eccentricity is dramatically reduced and can be eliminated during the analysis. With the prism a circle of radius $R$ will be transformed with a minor axis of $R \in$ and a major axis of $R$. It can be seen that for a prism with an apex angle of $90^{\circ}$ (right-angle prism), the resultant eccentricity is small. ${ }^{4}$

In the present ESPI system the object is located at distance $h$ from surface 1 of the prism. This gap laterally shifts the object beam with respect to the position of the reference beam. This shift, $\Delta x$, can be shown to be

$$
\Delta x=h \tan \theta_{3} .
$$

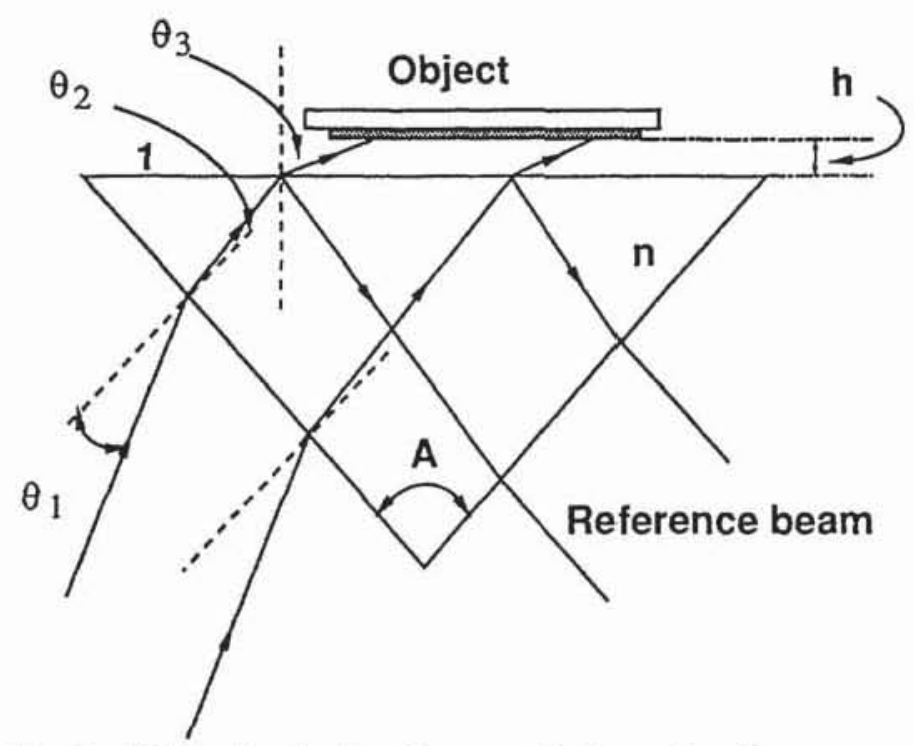

Fig. 2. Schematic showing the ray path through a three-corner prism. 
Exact overlap is obtained if the surface of the object under investigation is in contact with surface 1 of the prism. If $W$ is the width of the illuminating beam then the effective area of observation of the object is $(W-\Delta x)$. If the beam entering the prism is collimated then the object information over a region of $\Delta x$ is lost because of shear between the reference and object beam. However, for a converging beam entering the prism, the reference beam comes to focus before the focal plane of achromat $L_{2}$ subtending a larger cone angle than the object beam. Consequently, at the photosensor plane, the reference beam covers the entire image of the object. For large angles of illumination $\Delta x$ can sometimes be as large as $W$. Consequently, care must be taken to reduce $h$ to obtain as large an area of observation as possible. This problem has been solved through the use of a Dove prism instead of a right-angle prism. ${ }^{6}$ However, we propose a different method in which two identical prisms are used, as shown in Fig. 3. The first prism generates the reference and object illuminating beam, and the second prism is used to recombine them. Another advantage of this schematic arrangement is that phase stepping can be introduced in the reference beam. To maximize the effective area of observation, we can show that the distance at which the object should be placed is

$$
h \simeq \frac{H\left[1-\frac{\theta_{1}}{\sqrt{2}}+\frac{d}{\tan \left(45+\theta_{1}\right)}\right]}{\tan \theta_{3}}
$$

where $H$ is the height of each prism, the apex angle of each prism is $90^{\circ}$, and $d$ is the distance of the mirror from the top of the prism. We have derived Eq. (4) by assuming that $\theta_{1}$ is small, which is usually the case as the incident beam to the first surface of the prism is close to the normal. Angle $\theta_{1}$ is expressed in radians in Eq. (4). Depending on $H$, the angle of incidence of the beam, and $d$, the object can be placed at relatively large distances when compared with a single-prism geometry.

In the present configuration (Figs. 1 and 3) the reference and object beams travel almost identical

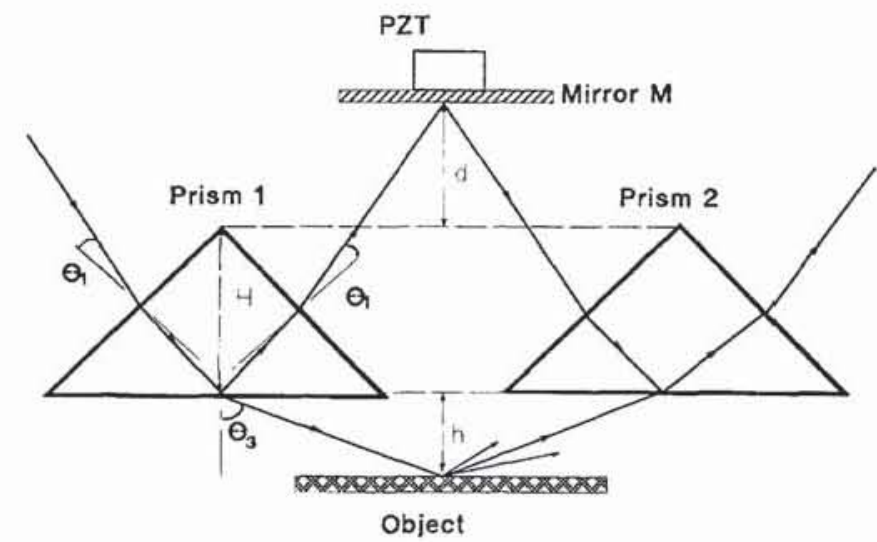

Fig. 3. Diagram showing the two-prism arrangement used for phase stepping. In this geometry the object can be placed at a reasonable distance from the prism.

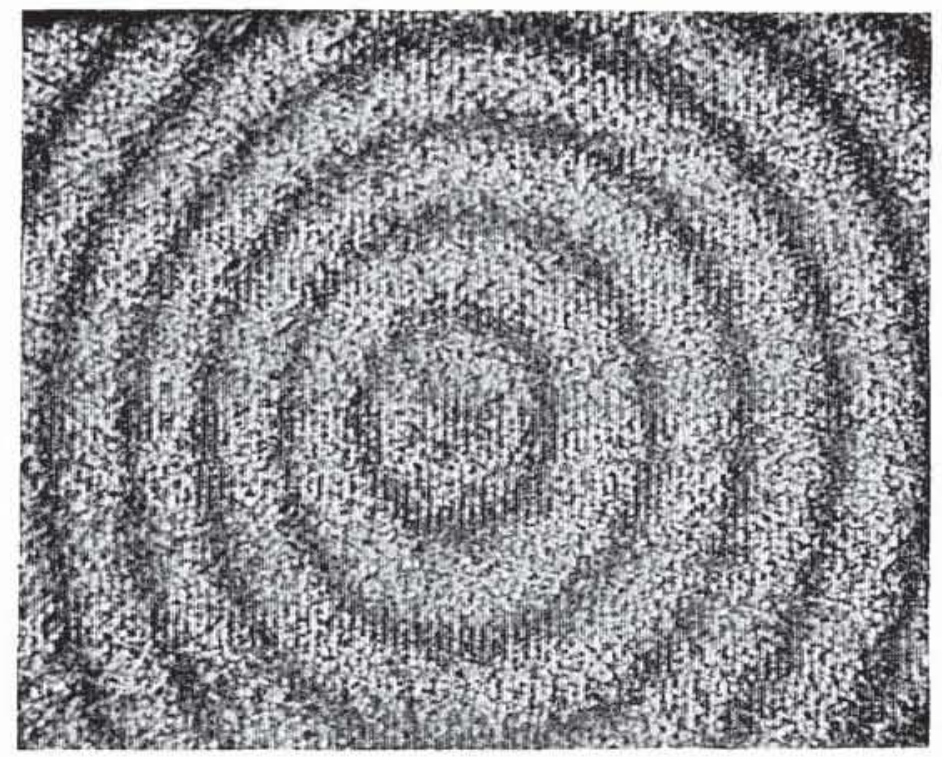

Fig. 4. Photograph of the fringes for a circular diaphragm clamped along the edges and loaded at the center. The angle of illumination was determined to be $76^{\circ}$, and the object was loaded to $10 \mu \mathrm{m}$.

paths, which is similar to the quasi-equal path ESPI system. ${ }^{9,10}$ The sensitivity of the method to the out-of-plane motion $w$ and in-plane motion $u$ is ${ }^{1}$

$$
\begin{aligned}
& w(x, y)=\frac{m \lambda}{\cos \alpha_{1}+\cos \alpha_{2}}, \\
& u(x, y)=\frac{m \lambda}{\sin \alpha_{1}-\sin \alpha_{2}},
\end{aligned}
$$

where $m$ is a positive integer, $\alpha_{1}$ is the angle of illumination of the object (this is $\theta_{3}$ in Fig. 3 ), $\alpha_{2}$ is the angle of observation, and $\lambda$ is the wavelength of light used. Because $\alpha_{1}=\alpha_{2}=\alpha$, the system is sensitive only to out-of-plane displacements and the sensitivity is reduced to $\lambda /(2 \cos \alpha)$. The sensitivity can be varied from $\lambda / 2$ to $8 \lambda$. To change the sensitivity of the interferometer, we rotate the prism and the object as a unit so that the angle of the beam entering the prism is altered.

In ESPI, and interferometry in general, quantitative evaluation is performed by the extraction of the phase information through the use of phase-stepping routines. In the right-angle prism of Fig. 1 the reference beam is generated from surface 1 , and therefore the phase of the reference beam cannot be stepped. In Fig. 3 the reference beam generated from the first prism is reflected to the second prism through the use of a mirror mounted on a piezoelectric translator $(\mathrm{PZT})$. We can now introduce a known amount of phase step in the reference beam by translating mirror M. The disadvantage of this scheme is the need for alignment of the reference and the object beams. However, one can achieve this by looking through the second prism and rotating it until the reference and object beams are collinear. 


\section{Experimental Results}

The green wavelength light $(\lambda=514 \mathrm{~nm})$ from an argon-ion laser was used for our experiments, and a right-angle prism as shown in Fig. 1 was used to obtain oblique angle of incidence and observation. The object of study was a circular diaphragm clamped along its edge and loaded at the center. The output signal from the CCD camera was processed on a FG 100 frame-grabber board in a host computer. Figure 4 shows the fringes obtained when the object was loaded to $10 \mu \mathrm{m}$ between exposures. The illumination angle $\left(\theta_{3}\right)$ was estimated to be around $76^{\circ}$. An area of the object of only $30 \mathrm{~mm} \times 30 \mathrm{~mm}$ was observed because of the size of the prism. Each fringe in the interferogram corresponds to a $1.1-\mu \mathrm{m}$ variation of the out-of-plane deformation.
The schematic arrangement in Fig. 3 was used to perform phase-stepping techniques. The small faces of the two identical prisms were $15 \mathrm{~mm} \times 15 \mathrm{~mm}$; therefore, the area of the object under investigation was relatively small. Figure 5 shows the results of phase stepping in this interferometer. The threebucket algorithm was used for the determination of the phase from the intensity patterns, and the fringes were low-pass filtered and smoothed with $5 \times 5$ and $7 \times 7$ windows. The object under investigation was a plane plate tilted between exposures. The angle of illumination in this case is $50^{\circ}$. The interferometer was used for nondestructive testing applications because the decreased sensitivity could be used to enhance the defect with large-object deformation. We created a defect behind the circular diaphragm by

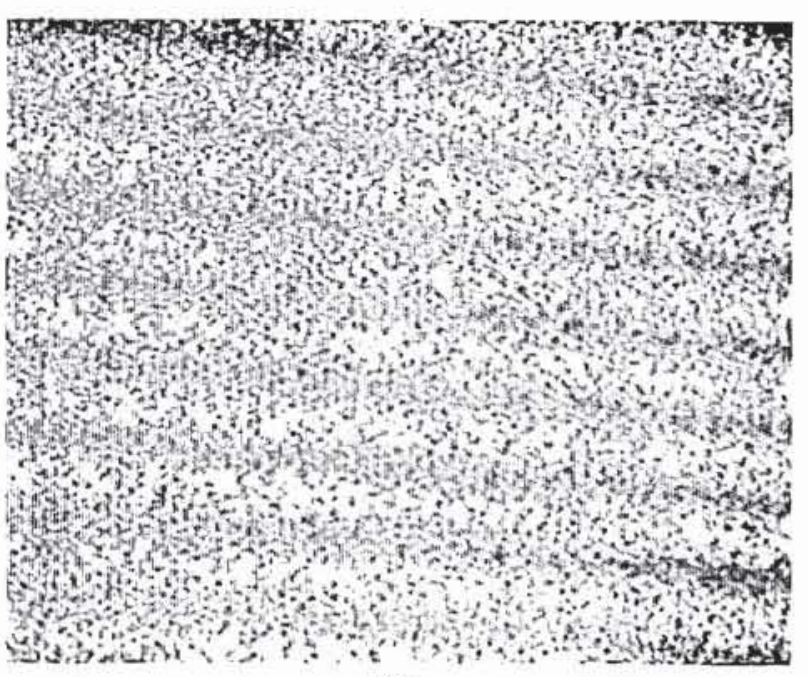

(a)

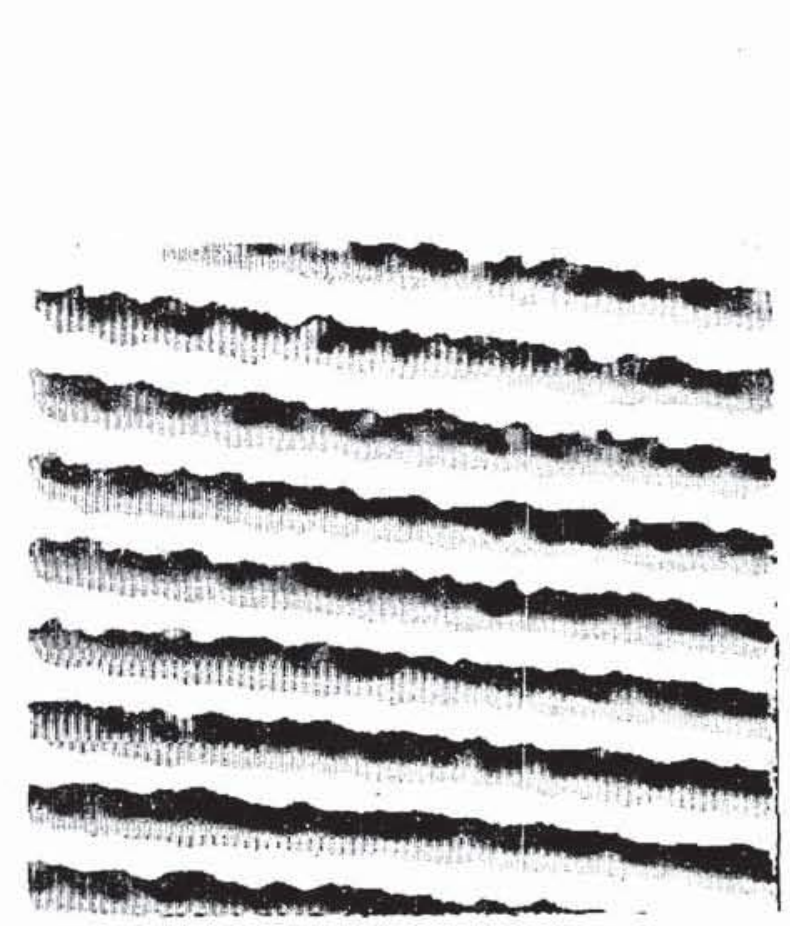

(b)

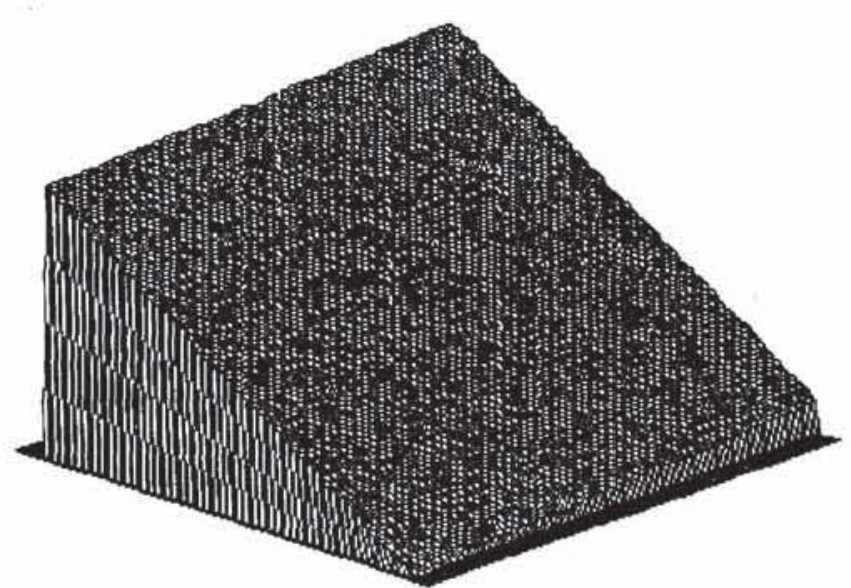

(c)
Fig. 5. Results of phase stepping in the oblique incidence interferometer. The object of study was a plate tilted between exposures: (a) the tilt fringes, (b) the phase map, (c) the three-dimensional plot of the surface tilt, (d) the contour maps obtained from the unwrapped phase map (peak to valley is $3.6 \mu \mathrm{m}$ ). 
milling out part of the material. Inconsistent results were obtained, and it was not easy for us to locate the defect site by spotting the deviation of the fringes. The experiments are under progress to enhance this nondestructive testing application.

\section{Conclusions}

We have demonstrated the principle and working of oblique incidence and observation in ESPI. One can change the sensitivity of the method by rotating the prism and the object. A method that uses two identical prisms was introduced in which the object can be placed at a reasonable distance from the prism. We accomplished phase stepping in this setup by shifting the reference beam with a PZT mirror. The disadvantages of this method are that (a) the prism size limits the area of the object under investigation and (b) the image of the object is aberrated, although this was reduced considerably by an aperture placed at the focal plane of the imaging lens. In contrast, the advantages of the method are (a) the experimental setup is simple, (b) it is easy to align, (c) a wide range of sensitivity can be introduced, between $\lambda / 2$ and $8 \lambda$, and (d) the reference beam in the modified setup can be phase shifted.

C. Joenathan thanks the Humboldt foundation for financial support. The authors also thank Mike Bartelt for fruitful discussion.

\section{References}

1. R. Jones and C. W. Wykes, Holographic and Speckle Interferometry (Cambridge U. Press, Cambridge, England, 1983), Chap. 4, pp. 183-188.

2. O. J. Lokberg, "The present and future of ESP," in Industrial Laser Interferometry, R. J. Pryputniewicz, ed., Proc. Soc. Photo-Opt. Instrum. Eng. 746, 86-97 (1987).

3. C. Joenathan, "Recent developments in electronic speckle pattern interferometry," in Proceedings of the SEM Conference, Milwaukee, WI 1991 (Society for Experimental Mechanics, Inc., Bethel, Conn., 1991), pp. 198-204.

4. N. Abramson, "The 'interferoscope', a new type of interferometer with variable fringe separation," Optik 30, 56-71 (1969).

5. K. G. Birch, "Oblique incidence interferometry applied to nonoptical surfaces,” J. Phys. E. 6, 1045-1048 (1973).

6. M.D.A. MacBean, "Oblique incidence interferometry of rough surfaces using a novel Dove-prism spectrometer," Appl. Opt. 23, 4024-4028 (1984).

7. D. Boebel, B. Packross, and H. J. Tiziani, "Phase shifting in an oblique incidence interferometer,” Opt. Eng. 30, 1910-1914 (1991).

8. C. Joenathan, "Effect of non-linearity of the TV camera in electronic speckle pattern interferometry," Optik 85, 33-37 (1990).

9. S. Peng, C. Joenathan, and B. M. Khorana, "Quasi-equal path electronic speckle pattern interferometric system," Opt. Lett. 17, 1040-1042 (1992).

10. C. Joenathan and B. M. Khorana, "Modified quasi-equal path ESPI system,” Appl. Opt. 32, 5724-5726 (1993). 